\title{
Early reperfusion strategy improves the outcomes of surgery for type A acute aortic dissection with malperfusion
}

\author{
Keiji Uchida, MD, PhD, Norihisa Karube, MD, PhD, Keiichiro Kasama, MD, \\ Tomokazu Minami, MD, PhD, Shota Yasuda, MD, PhD, Motohiko Goda, MD, PhD, \\ Shinichi Suzuki, MD, PhD, Kiyotaka Imoto, MD, PhD, and Munetaka Masuda, MD, PhD
}

\section{ABSTRACT}

Objective: The control of malperfusion is the key to improving the outcomes of surgery for type A acute aortic dissection. We revised our treatment strategy to reperfuse each ischemic organ before central repair.

Methods: Our current early reperfusion strategy consists of percutaneous coronary artery intervention for coronary malperfusion, direct surgical fenestration for carotid artery occlusion, active perfusion of the superior mesenteric artery for visceral malperfusion, and external shunting from the brachial artery to the femoral artery for lower limb ischemia. Central repair is performed without delay after reperfusion therapy, but if irreversible organ damage is recognized, further aggressive treatment is discontinued.

Results: Among 438 patients who underwent initial treatment for type A acute aortic dissection, malperfusion in one or more organs was diagnosed in 108 patients $(24 \%)$. We applied an early reperfusion strategy in 33 patients, (coronary, 14 patients; carotid, 4; visceral, 7; lower extremity, 8). Central repair was then performed in 28 patients. One patient $(3.6 \%)$ died of pneumonia; 27 patients overcame the ischemic organ damage and survived. Among the 108 patients with malperfusion, 10 patients $(9.3 \%)$ were treated medically without early reperfusion and central repair. During the same period, mortality from central repair procedures in patients with malperfusion who had not received early reperfusion therapy was 12 of $65(18 \%)$, and the mortality of patients without malperfusion was 9 of $262(3.4 \%)$. Malperfusion was a serious risk factor for hospital death, but the mortality rate of the patients with an early reperfusion strategy was significantly $(P<.01)$ lower than the patients without early reperfusion.

Conclusions: Our strategy might improve the outcomes of surgery for type A acute aortic dissection with malperfusion. This strategy enables us to avoid unproductive central repair procedures in irreversibly damaged patients. (J Thorac Cardiovasc Surg 2018;156:483-9)

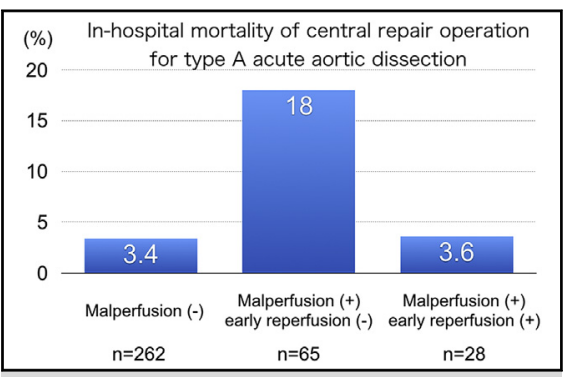

In-hospital mortality of central repair surgery for type A acute aortic dissection.

\section{Central Message}

Early reperfusion strategy improves the outcomes of surgery for type A acute aortic dissection with malperfusion, and enables us to avoid unproductive surgeries in irreversibly damaged patients.

\section{Perspective}

How to control malperfusion is the key to improving the outcomes of surgery for type A acute aortic dissection. Immediate aortic repair and primary entry-tear resection is mandatory, but seems to be suboptimal. We established early reperfusion techniques, especially coronary, visceral, and lower limb. The mortality rate of subsequent aortic surgery has decreased.

See Editorial Commentary page 490.

See Editorial page 469.
How to control malperfusion is the key to improving the outcomes of surgery for type A acute aortic dissection. Immediate central aortic repair and primary-entry tear

\footnotetext{
From the Cardiovascular Center, Yokohama City University Medical Center, Yokohama, Japan.

Read at the 97th Annual Meeting of The American Association for Thoracic Surgery, Boston, Massachusetts, April 29-May 3, 2017.

Received for publication April 30, 2017; revisions received Jan 3, 2018; accepted for publication Feb 2, 2018; available ahead of print March 13, 2018.

Address for reprints: Keiji Uchida, MD, PhD, Cardiovascular Center, Yokohama City University Medical Center, 4-57 Urafune-cho, Minami-ku, Yokohama 232-0024, Japan (E-mail: k_uchida@yokohama-cu.ac.jp).

$0022-5223 / \$ 36.00$

Copyright (c) 2018 by The American Association for Thoracic Surgery https://doi.org/10.1016/j.jtcvs.2018.02.007
}

resection is mandatory, and resolves most cases of dynamic type obstruction. ${ }^{1,2}$ However, acute occlusion of the coronary, carotid, or visceral arteries sometimes leads to irreversible organ damage after aortic surgery. ${ }^{3-5} \mathrm{We}$ revised our treatment strategy to directly reperfuse each ischemic organ before central repair (Video 1). We report our treatment strategy and its results.

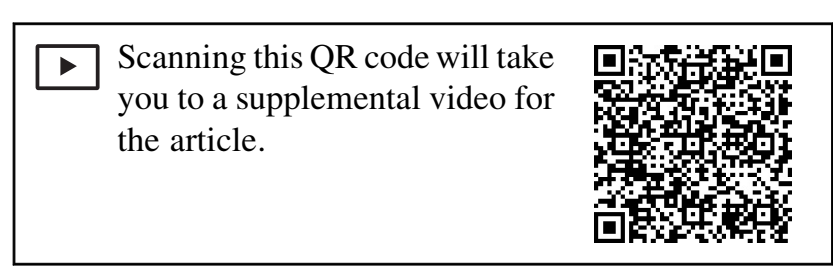




\section{Abbreviations and Acronyms \\ $\mathrm{AR}=$ aortic regurgitation \\ $\mathrm{CT}=$ computed tomography \\ $\mathrm{ER}=$ early reperfusion \\ $\mathrm{MP}=$ malperfusion}

\section{METHODS}

The subjects were 438 patients with Stanford type A acute aortic dissection who underwent initial treatment in our hospital between 2006 and 2016. Emergency surgery was indicated for all patients with type A acute aortic dissection, including the acute thrombosed type. For diagnosis, contrast-enhanced computed tomography (CT) was primarily performed, and transesophageal echography was performed in all patients who underwent surgery. Malperfusion was diagnosed in patients who had clinical symptoms as well as occlusion of the corresponding arteries on diagnostic imaging. This study was approved by the institutional ethics committee/review board of Yokohama City University, which waived the requirement for informed patient consent because of the retrospective nature of this study (date and number of institutional review board approval November 16, 2017; B171000049).

Our basic surgical strategies of central repair operations for type A acute aortic dissection are as follows. As arterial lines for cardiopulmonary bypass, side branches of the axillary and femoral arteries were used. The left or right side was selected on the basis of CT findings, and the absence of true-lumen collapse was always confirmed on transesophageal or epiaortic echography at the start of extracorporeal circulation. For brain protection, anterograde selective cerebral perfusion was performed. The extent of aortic replacement was determined on the basis of the entry site. If the entry was located in the ascending aorta or the lesser curvature side of the arch, ascending aorta or hemi-arch replacement was performed. If the entry was located in the greater curvature side of the arch or in the proximal descending aorta, total arch replacement was conducted. If there was no entry from the ascending aorta to the proximal descending aorta, we replaced only the ascending aorta or performed entry closure using stent graft insertion.

The diagnostic criteria for malperfusion and current treatment strategies for each ischemic organ are described in the following sections. Renal arteries are often involved in dissection, but their CT findings sometimes vary depending on the time delay after contrast medium injection, making it difficult to judge whether they are occluded or not. Unilateral renal malperfusion rarely causes any clinical symptoms. We excluded patients with renal malperfusion from our study.

\section{Coronary Malperfusion}

Patients with ST elevation on a 12-lead electrocardiogram associated with wall hypokinesis at the corresponding region on echocardiography were considered to have coronary artery ischemia. After the administration of heparin, emergency percutaneous coronary intervention was urgently performed at the same time as starting to prepare the operation room. We did not use antiplatelet drugs such as aspirin or clopidogrel. Intravascular ultrasonography of the coronary arteries was performed to confirm the extent of dissection and the luminal diameter of the coronary arteries. A stent was then placed (Figure 1). Patients whose cardiac function improved after successful coronary artery reperfusion were transferred to the operating room to undergo a central repair procedure. When a stent was placed in the left main trunk, prophylactic bypass grafting to the left anterior descending coronary artery using the saphenous vein graft was performed because fatal, subacute stent thrombosis might occur after surgery without antiplatelet therapy.

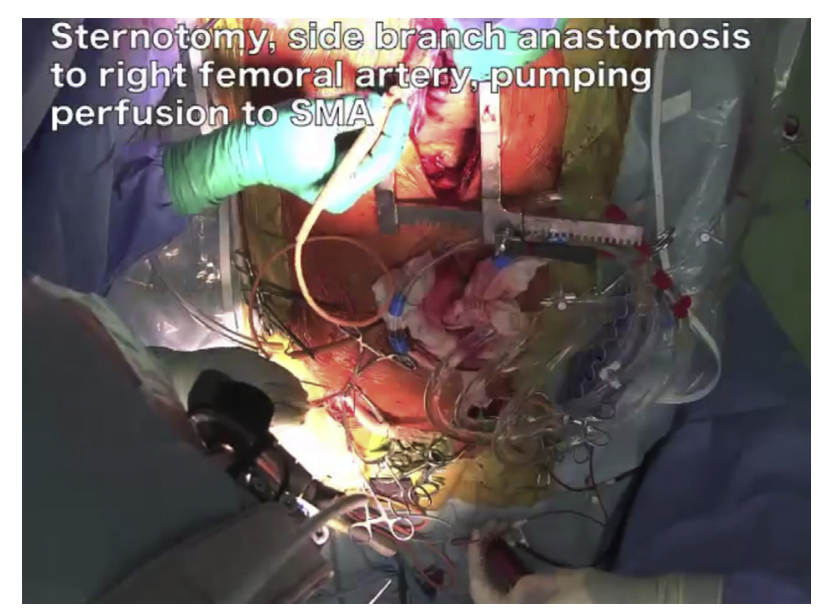

VIDEO 1. Left main stenting for coronary malperfusion, superior mesenteric artery cannulation and perfusion for visceral malperfusion, and carotid artery fenestration for brain malperfusion and coma. Video available at: http://www.jtcvsonline.org/article/S0022-5223(18)30355-6/fulltext.

\section{Brain Malperfusion}

Patients with disturbed consciousness or paralysis unaccompanied by circulatory collapse in whom carotid artery dissection was seen on CT or ultrasonography were considered to have brain malperfusion. We did not perform central repair for comatose patients and the patients in whom large cerebral infarction was already evolved. In the other patients, central repair is primarily performed without early reperfusion strategy. ${ }^{6}$ In ultra-acute comatose patients with carotid artery occlusion, several types of reperfusion therapy, such as direct surgical fenestration of the carotid artery (Figure 2), direct perfusion of the brachiocephalic artery after median sternotomy, and catheter intervention were performed depending on the attending surgeon's decision.

\section{Spinal Cord Malperfusion}

Patients with paraplegia and paraparesis that were not caused by impaired blood flow of the lower extremities were considered to have spinal cord malperfusion. In patients with impaired blood flow of the lower extremities, the results of pinprick tests at the level of Th10 were referred to at the time of diagnosis. Because static occlusion of intercostal arteries is a potential cause, reperfusion therapy cannot initially be performed. Some aged patients in whom false lumen of ascending aorta had thrombosed, underwent only spinal drainage without performing the central repair procedure for dissection.

\section{Visceral Malperfusion}

Patients with abdominal pain and occlusion of the celiac artery, the superior mesenteric artery, or both on CT imaging were considered to have visceral malperfusion. In patients with disturbed consciousness, the presence of abnormal diarrhea with mucous and bloody stools was referred to in diagnosis. Laparotomy was performed before central repair. The retroperitoneum was incised to expose the main trunk and the branches of the superior mesenteric artery. Direct echography is an effective means of identifying a pulseless superior mesenteric artery. Branches without dissection were cut down. An 8-French soft flexible tube was inserted toward the center of the abdominal aorta and was withdrawn while measuring intraluminal pressure to confirm the pressure drop in the superior mesenteric artery. The superior mesenteric artery was then perfused by actively pumping arterial blood via a soft flexible tube from the femoral artery sheath, performed with the use of a 50-mL syringe (Figure 3). ${ }^{7}$ Atonic small intestine began peristaltic movement. While continuing active perfusion, the central repair 


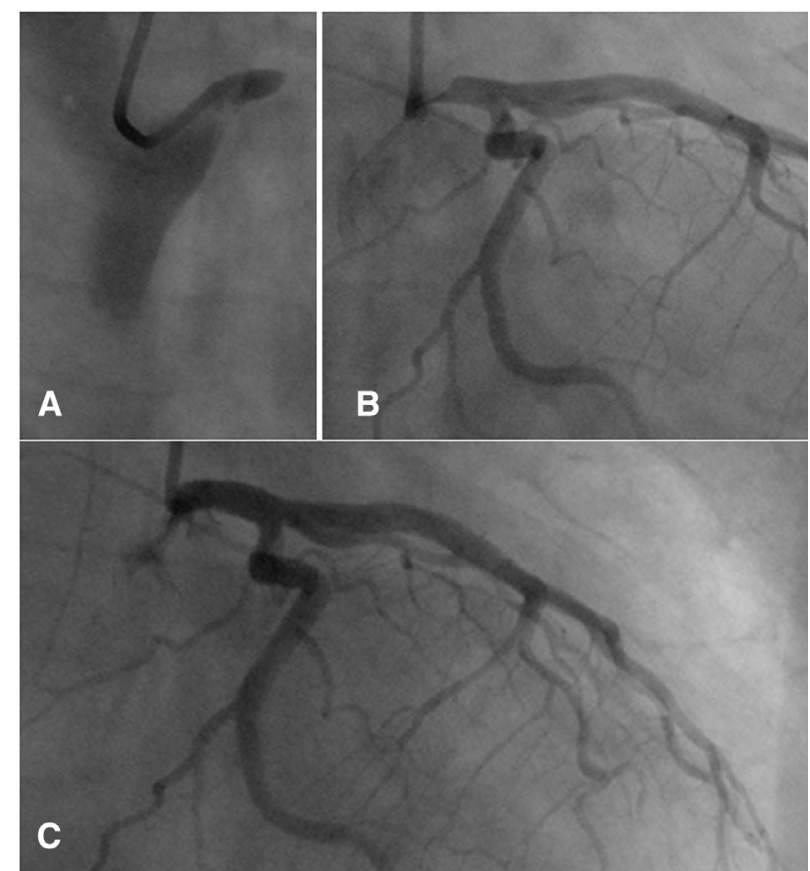

FIGURE 1. Percutaneous coronary intervention for the patients with coronary malperfusion. A, Left coronary angiography from pseudo lumen. B, Left coronary angiography from true lumen. C, After stenting for left main trunk.

procedure was started. After the central repair, normalization of the superior mesenteric arterial pressure should be confirmed. Then the soft flexible tube was removed, superior mesenteric artery was sutured, and the abdomen was closed.

\section{Lower Limb Malperfusion}

The presence of ischemic pain of a lower limb, disappearance of the femoral artery pulse, and iliac artery occlusion on CT imaging were considered to indicate lower limb malperfusion. If time is required to prepare to perform the central repair procedure and severe pain exists,

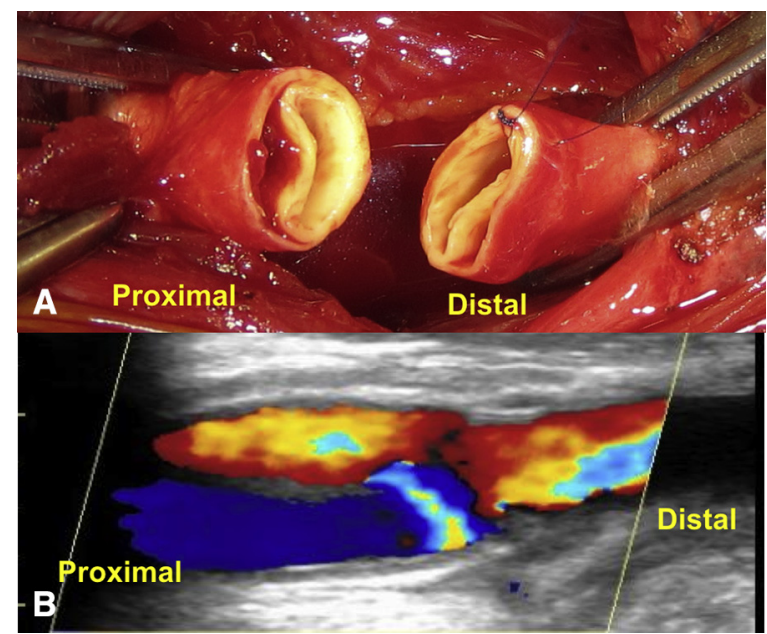

FIGURE 2. Direct fenestration of carotid artery for the patients with brain malperfusion. A, Intraoperative finding. B, Postoperative echography.

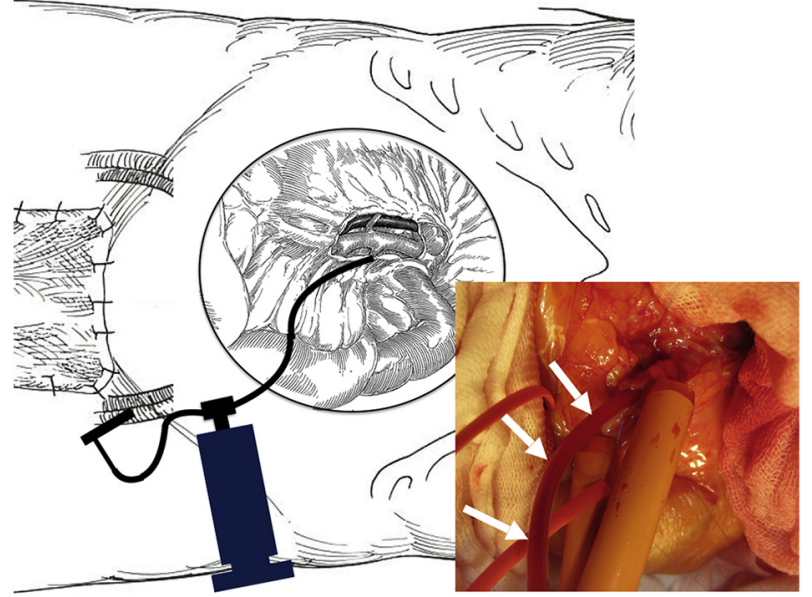

FIGURE 3. Active perfusion of superior mesenteric artery for the patients with visceral malperfusion. An 8-French soft flexible tube is inserted to the branch of the superior mesenteric artery and connected to the femoral artery sheath.

6-French arterial sheaths are inserted into the brachial artery and the affected side of the femoral artery and connected (Figure 4). This serves as an external shunt. We usually perform echography to puncture the pulseless femoral artery. In the operating room, a short vascular graft was anastomosed to ischemic femoral artery and was used as the arterial line of the heart-lung machine.

\section{RESULTS}

The mean age of the 438 patients (228 men and 210 women) was $66 \pm 13$ years. Communicating aortic dissection was present in 301 patients $(69 \%)$, thrombosed-type aortic dissection in 137 patients $(31 \%),{ }^{8}$ pericardial effusion in 199 patients $(45 \%)$, and cardiac tamponade in 118 patients $(27 \%)$. Before admission, 14 patients $(3 \%)$ had cardiopulmonary arrest and required resuscitation.

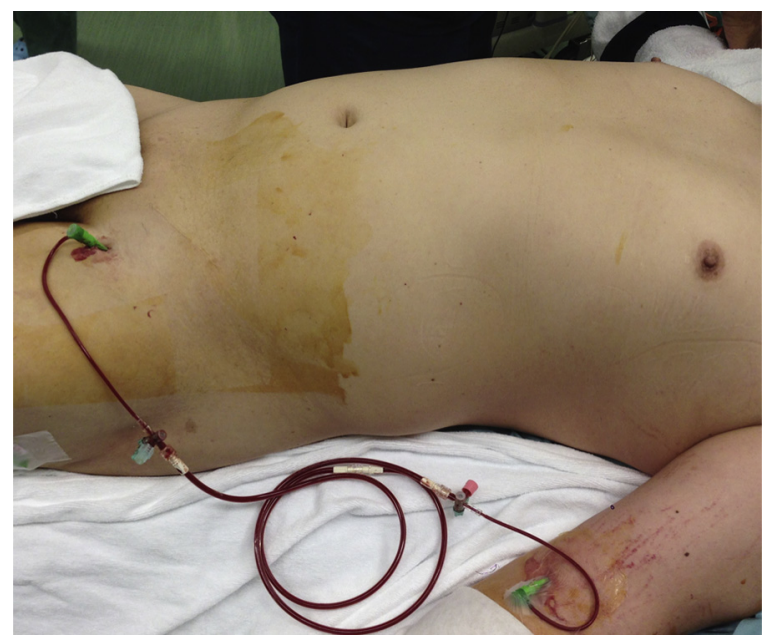

FIGURE 4. External shunt is established for the patient with lower limb malperfusion. 
TABLE 1. Differences in demographic characteristics according to the presence or absence of malperfusion in 438 patients

\begin{tabular}{lccc}
\hline \multicolumn{1}{c}{ Characteristic } & MP present & MP absent & $\boldsymbol{P}$ \\
\hline Number of patients & 108 & 330 & \\
Age older than 70 y & $34(31)$ & $162(49)$ & .001 \\
Male sex & $63(58)$ & $165(50)$ & .132 \\
Patent false lumen & $103(95)$ & $198(60)$ & .000 \\
Cardiac tamponade & $21(19)$ & $97(29)$ & .043 \\
AR > moderate & $36(33)$ & $75(23)$ & .088 \\
Central repair & $93(86)$ & $263(80)$ & .138 \\
In-hospital death & $24(22)$ & $30(9)$ & .000 \\
\hline
\end{tabular}

$M P$, Malperfusion; $A R$, aortic regurgitation.

Moderate or severe aortic regurgitation was present in 111 patients $(25 \%)$.

Malperfusion was found in 108 patients $(24 \%)$ and involved the coronary arteries in 25 patients $(5.7 \%)$, the brain in 36 patients $(8.2 \%)$, the spinal cord in 6 patients $(1.4 \%)$, the viscera in 18 patients $(4.1 \%)$, and the lower limbs in 48 patients $(11 \%)$. When patients with malperfusion were compared with patients without malperfusion, the malperfusion group was found to have a significantly higher number of younger patients with communicating dissection and a lower incidence of cardiac tamponade. Treatment outcomes were significantly poorer in the malperfusion group (24 deaths; mortality rate, $22 \%$ ) than in the group without malperfusion (30 deaths; mortality rate, $9 \%$; Table 1). Early reperfusion therapy was given to 33 patients with malperfusion, for the coronary in 14 patients, for the brain in 4 patients, for the viscera in 7 patients, and for the lower extremities in 8 patients.

Because of factors such as advanced age, thrombosedtype dissection in which time had elapsed from the onset, severe comorbidity, and irreversible organ damage caused by malperfusion, central repair was not performed in 83 $(19 \%)$ of the 438 patients, 15 patients $(3 \%)$ with malperfusion and 68 patients $(16 \%)$ without malperfusion. Among 33 patients who had received early reperfusion therapy, central repair was not performed in 5 patients because of the presence of myocardial or brain dysfunction (Table 2).

Central repair was performed in 355 patients (187 men and 168 women). The mean age was $64 \pm 12$ years. Stent graft entry closure was performed in 8 patients with retrograde type A aortic dissection. Open surgery was performed in the other 347 patients. Intraoperatively, an entry tear was found in the ascending aorta in 161 patients $(45 \%)$ and the aortic arch in 96 patients $(27 \%)$. The aortic root procedure was supracoronary replacement in 307 patients, root replacement in 24 , reimplantation in 13 , and aortic valve replacement in 3. Ascending aortic replacement including hemiarch repair was performed in 249 patients, and total arch replacement was performed in 98 . As for surgical outcomes, 22 patients $(6.2 \%)$ died during hospitalization, 39 patients $(11 \%)$ had cerebral infarction, and 7 patients $(2 \%)$ had spinal cord infarction. Patients who underwent central repair were classified into 3 groups to compare outcomes: 262 patients without malperfusion, 28 patients with malperfusion who underwent early reperfusion, and 65 patients with malperfusion who did not undergo early reperfusion (Table 3). The mortality rate was significantly higher in the patients with malperfusion who did not undergo early reperfusion $(18 \%)$ than in other groups. In contrast, similar good outcomes were obtained in the patients with malperfusion who underwent early reperfusion and in the patients without malperfusion. Among the 28 patients with malperfusion who underwent early reperfusion, only 1 patient $(3.6 \%)$ with lower limb ischemia and severe emphysema died of pneumonia and sepsis. The other 27 patients overcame the ischemic organ damage and survived.

Coronary malperfusion occurred in 25 patients. The left coronary artery was involved in 13 patients and right coronary artery in 12 patients. Nine patients were in shock, with a systolic blood pressure of $90 \mathrm{~mm} \mathrm{Hg}$ or less. Fourteen patients underwent early reperfusion therapy, 7 of them had a diagnosis of acute myocardial infarction, without realizing the presence of acute aortic dissection. Among the 14 patients who could undergo early coronary reperfusion, 11 patients underwent central repair, and 2 of them temporally required extracorporeal membrane oxygenation. All of these patients survived. Among the 11 patients in whom coronary reperfusion could not be performed preoperatively, 9 patients underwent central repair, and 5 patients could not be weaned from cardiopulmonary bypass and required extracorporeal membrane oxygenation. Five patients died during hospitalization; the surgery mortality rate was $56 \%$.

Brain malperfusion occurred in 36 patients: 11 had coma, 15 had hemiplegia, and 10 had transient ischemic attacks. Reperfusion therapy was initially performed in 4 patients. One patient underwent surgical fenestration of the carotid artery, and 1 underwent external shunting from the femoral

TABLE 2. Patient flow diagram and mortality in each group

\begin{tabular}{|c|c|c|c|c|c|c|}
\hline \multicolumn{7}{|c|}{ Type $A$ acute aortic dissection $(n=438)$} \\
\hline \multirow[b]{3}{*}{ Central repair } & \multicolumn{4}{|c|}{ Malperfusion $(n=108)$} & \multirow{2}{*}{\multicolumn{2}{|c|}{ No malperfusion $(\mathbf{n}=\mathbf{3 3 0})$}} \\
\hline & \multicolumn{2}{|c|}{ Early reperfusion $(n=33)$} & \multicolumn{2}{|c|}{ No early reperfusion $(n=75)$} & & \\
\hline & Yes $(n=28)$ & No $(n=5)$ & Yes $(n=65)$ & No $(\mathbf{n}=10)$ & Yes $(n=262)$ & No $(n=68)$ \\
\hline Mortality, n (\%) & $1(3.6)$ & $4(80)$ & $12(19)$ & $7(70)$ & $9(3.4)$ & $21(31)$ \\
\hline
\end{tabular}


TABLE 3. Differences in demographic characteristics according to the presence or absence of malperfusion and early reperfusion in 355 patients who underwent central repair

\begin{tabular}{|c|c|c|c|c|}
\hline & MP present, ER present & MP present, ER absent & MP absent & $\boldsymbol{P}$ \\
\hline Number of patients & 28 & 65 & 262 & \\
\hline Age older than $70 y$ & $8(29)$ & $19(29)$ & $109(42)$ & .101 \\
\hline Male sex & $18(64)$ & $38(58)$ & $131(50)$ & .208 \\
\hline Thrombosed false lumen & $0(0)$ & $4(6.2)$ & $87(33)$ & .000 \\
\hline Cardiac tamponade & $3(11)$ & $13(20)$ & $78(30)$ & .040 \\
\hline $\mathrm{AR}>$ moderate & $12(43)$ & $23(35)$ & $75(29)$ & .276 \\
\hline Preoperative CPA & $0(0)$ & $2(3.1)$ & $7(2.7)$ & .662 \\
\hline Entry in ascending aorta and arch & $24(86)$ & $50(77)$ & $183(70)$ & .135 \\
\hline Root surgery & $7(25)$ & $3(4.6)$ & $27(10)$ & .013 \\
\hline Total arch replacement & $11(39)$ & $21(32)$ & $66(25)$ & .183 \\
\hline Stroke and/or paraplegia & $4(14)$ & $19(29)$ & $23(8.8)$ & .000 \\
\hline In-hospital death & $1(3.6)$ & $12(18)$ & $9(3.4)$ & .000 \\
\hline
\end{tabular}

Data are presented as $\mathrm{n}(\%)$ except where otherwise noted. $M P$, Malperfusion; $E R$, early reperfusion; $A R$, aortic regurgitation; $C P A$, cardiopulmonary arrest.

artery to the brachial artery because of brachiocephalic artery occlusion. Coma did not improve in these 2 patients, and central repair was not achieved. Left common carotid artery occlusion by intimal intussusception of the aortic arch was improved by catheter maneuver in 1 patient. The other patient underwent direct brachiocephalic artery perfusion after median sternotomy. Central repair was performed in 25 patients, including the later 2 patients, among whom 5 patients $(20 \%)$ died, and $12(48 \%)$ had cerebral infarction.

Among 6 patients with paraplegia, 2 patients had thrombosed false lumen and therefore underwent spinal drainage without performing central repair. The paraplegia improved, and the patients could walk. The other 4 patients underwent central repair. There were no deaths, but paraplegia persisted after surgery.

Among the 18 patients with visceral malperfusion, 5 patients initially underwent central repair, and 2 patients died of intestinal necrosis. Among the 13 patients who underwent exploratory laparotomy, 7 patients had critical ischemia of the intestine and underwent superior mesenteric artery perfusion and central repair. All of these patients survived. Six patients were judged to have no ischemia in the small intestine and underwent central repair. Three of these patients had severe necrotic pancreatitis after surgery, and 1 patient died.

Among the 48 patients with lower extremity ischemia, 8 had bilateral ischemia, 20 had right lower extremity ischemia, and 20 had left lower extremity ischemia. Eight patients underwent shunting of the brachial artery and the femoral artery, and their symptoms promptly improved. All patients underwent central repair. Eight patients $(17 \%)$ died. The main cause of death was ischemia of other organs. No patient had myonephrotic metabolic syndrome.

\section{DISCUSSION}

The results of surgery for Stanford type A acute aortic dissection have improved. The mortality rate was $10.6 \%$ according to the annual report of the Japanese Association for Thoracic Surgery. ${ }^{9}$ Currently, the most serious complication affecting outcomes is malperfusion. Theoretically, a prompt central repair procedure with entry tear resection would improve the dynamic malperfusion. However, mortality remains high in such patients. ${ }^{1-5}$ The permissive ischemic time (ie, the acceptable ischemic time before reperfusion) is considered to be several minutes in the brain, 90 minutes in the coronary arteries, 3 hours in the abdomen, and 3 to 4 hours in the lower extremities. ${ }^{10}$ In some patients who initially undergo central repair, there might not be adequate time to salvage the ischemic organs.

The mortality rate of percutaneous coronary intervention for acute myocardial infarction due to unprotected left main coronary artery occlusion was reported to be $11 \%$ to $44 \% .{ }^{11}$ Good outcomes are thus unlikely to be achieved by reperfusion induced by central repair. We collaborated with cardiologists on percutaneous coronary intervention of the patient with coronary malperfusion, and reported the successful outcomes. ${ }^{12,13}$ Since then, we have extended our early reperfusion strategy to patients with abdominal ischemia and lower extremity ischemia. CT image interpretation, ability of diagnosis, and various reperfusion techniques were gradually established.

In our study, patients who initially underwent early reperfusion had good outcomes, generally similar to those of patients without malperfusion. In particular, the outcomes of patients with myocardial ischemia and patients with abdominal ischemia have markedly improved, compared with previous results. ${ }^{14,15}$ However, in patients with a 
diagnosis of visceral malperfusion who underwent exploratory laparotomy, but had no decrease in blood flow in the superior mesenteric artery, severe pancreatitis frequently developed. In such patients, intervention into the celiac artery might be necessary.

The situation was quite different regarding brain malperfusion. The brain can endure complete ischemia for only a few minutes. If the carotid artery is occluded at the same time as the onset of aortic dissection, and no collateral circulation is present, no treatment is available to protect the brain. Central repair should be performed in patients in whom blood flow is somehow maintained because of spontaneous recanalization or collateral circulation through the circle of Willis. Consequently, reperfusion therapy administered after admission can improve outcomes in few patients.

Initially performing reperfusion therapy slightly delays the time to central repair. It cannot be ruled out that such a delay might increase the risk of cardiac tamponade. The time required for reperfusion has a learning curve. Recent patients require only 30 minutes for coronary stenting and for superior mesenteric artery perfusion. Furthermore, the incidence of cardiac tamponade was significantly lower in patients with malperfusion, than that in patients without malperfusion. ${ }^{8,16}$ In our study, no patient died of cardiac tamponade during the period required to perform initial reperfusion therapy. Therefore, the consequent delay in central repair was considered acceptable.

Not only early reperfusion therapy, but also selection of the cardiopulmonary bypass route has an important role in improving the outcomes of patients with malperfusion. Recent studies reported the ease and usefulness of transapical aortic cannulation and ascending aortic cannulation. ${ }^{17,18}$ In such cannulation, however, malperfusion might persist after starting cardiopulmonary bypass. Arterial flow via axillary and femoral artery might improve dynamic-type malperfusion associated with an entry in the ascending arch aorta. Lower limb malperfusion can be treated with bidirectional blood transmission via a side branch. The bloodflow route should be decided on the basis of detailed preoperative CT scans, and should be confirmed using intraoperative transesophageal echography. ${ }^{19}$

Patients who have acute aortic dissection with malperfusion are not necessarily directly transported to a hospital within a minimum time. The question arises whether the irreversibility of ischemic organs can be determined solely on the basis of the time that has elapsed? Malperfusion can occur several hours after the onset of dissection. Malperfusion might also be associated with repeated episodes of spontaneous reperfusion and reocclusion. The time from the onset of dissection should not be the sole reason for not performing surgery. However, central repair requires considerable effort, high costs, and much time. If only reperfusion of ischemic organs is performed before central repair, we can evaluate whether the patient's life can be saved or not. Central repair should not be performed if there is no improvement in cardiac function after inserting a stent into the left main trunk, if coma does not improve even after carotid artery fenestration, or if extensive necrosis of the small intestine is seen at the time of exploratory laparotomy for abdominal ischemia.

Because this was a retrospective observational study of the past 11 years, the early reperfusion strategy had been applied in only $31 \%$ of the patients who suffered malperfusion. We cannot prove the effectiveness of this strategy because the patients were not randomized and their backgrounds were different. However, their better outcomes than in previous reports made us believe that it might be a promising treatment option for this dreadful complication of acute aortic dissection.

\section{CONCLUSIONS}

This was a retrospective, historical study, and the number of patients with malperfusion was limited. However, we believe that our strategy might improve the outcomes of patients who undergo surgery for type A acute aortic dissection with malperfusion. This strategy also allows us to avoid unproductive central repair procedures in irreversibly damaged patients.

\section{Conflict of Interest Statement}

Authors have nothing to disclose with regard to commercial support.

\section{References}

1. Girardi LN, Krieger KH, Lee LY, Mack CA, Tortolani AJ, Isom OW. Management strategies for type A dissection complicated by peripheral vascular malperfusion. Ann Thorac Surg. 2004;77:1309-14.

2. Geirsson A, Szeto WY, Pochettino A, McGarvey ML, Keane MG, Woo YJ, et al. Significance of malperfusion syndromes prior to contemporary surgical repair for acute type A dissection: outcomes and need for additional revascularizations. Eur J Cardiothorac Surg. 2007;32:255-62.

3. Pacini D, Leone A, Belotti LM, Fortuna D, Gabbieri D, Zussa C, et al. Acute type A aortic dissection: significance of multiorgan malperfusion. Eur J Cardiothorac Surg. 2013;43:820-6.

4. Czerny M, Schoenhoff F, Etz C, Englberger L, Khaladj N, Zierer A, et al. The impact of pre-operative malperfusion on outcome in acute type A aortic dissection. J Am Coll Cardiol. 2015;65:2628-35.

5. Kamman AV, Yang B, Kim KM, Williams DM, Deeb GM, Patel HJ. Visceral malperfusion in aortic dissection: the Michigan experience. Semin Thorac Cardiovasc Surg. 2017:29:173-8.

6. Tsukube T, Haraguchi T, Okada Y, Matsukawa R, Kozawa S, Ogawa K, et al. Long-term outcomes after immediate aortic repair for acute type A aortic dissection complicated by coma. J Thorac Cardiovasc Surg. 2014;148:1013-8.

7. Okada Y, Okimoto M, Katsumata M, Takeuchi S. Temporary perfusion for mesenteric ischemia with acute type A aortic dissection. Ann Thorac Surg. 2007;83:293-4.

8. Uchida K, Imoto K, Karube N, Minami T, Cho T, Goda M, et al. Intramural hematoma should be referred to as thrombosed-type aortic dissection. Eur J Cardiothorac Surg. 2013;44:366-9; discussion 369.

9. Masuda M, Okumura M, Doki Y, Endo S, Hirata Y, Kobayashi J, et al. Thoracic and cardiovascular surgery in Japan during 2014 annual report by the Japanese Association for Thoracic Surgery. Gen Thorac Cardiovasc Surg. 2016;64: 665-97.

10. Francone M, Bucciarelli-Ducci C, Carbone I, Canali E, Scardala R, Calabrese FA, et al. Impact of primary coronary angioplasty delay on myocardial salvage, infarct size, and microvascular damage in patients with ST-segment 
elevation myocardial infarction: insight from cardiovascular magnetic resonance. J Am Coll Cardiol. 2009;54:2145-53.

11. Michael S, Dahodwala MQ. Percutaneous coronary intervention for acute myocardial infarction due to unprotected left main coronary artery occlusion. Catheter Cardiovasc Interv. 2015;85:416-20.

12. Imoto K, Uchida K, Suzuki S, Isoda S, Karube N, Kimura K. Stenting of a left main coronary artery dissection and stent-graft implantation for acute type A aortic dissection. J Endovasc Ther. 2005;12:258-61.

13. Imoto K, Uchida K, Karube N, Yasutsune T, Cho T, Kimura K, et al. Risk analysis and improvement of strategies in patients who have acute type A aortic dissection with malperfusion. Eur J Cardiovasc Surg. 2013;44:419-24.

14. Kawahito K, Adachi H, Murata S, Yamaguchi A, Ino T. Coronary malperfusion due to type A aortic dissection: mechanism and surgical management. Ann Thorac Surg. 2003;76:1471-6.

15. Eusanio MD, Trimarchi S, Patel HJ, Hutchison S, Suzuki T, Peterson MD, et al. Clinical presentation, management, and short-term outcome of patients with type A acute dissection complicated by mesenteric malperfusion: observations from the International Registry of Acute Aortic Dissection. J Thorac Cardiovasc Surg. 2013;145:385-90.
16. Uchida K, Imoto K, Takahashi M, Suzuki S, Isoda S, Sugiyama M, et al. Pathologic characteristics and surgical indications of superacute type A intramural hematoma. Ann Thorac Surg. 2005;79:1518-21.

17. Inoue Y, Takahasgi R, Ueda T, Yozu R. Synchronized epiaortic two-dimensiona and color Doppler echocardiographic guidance enables routine ascending aortic cannulation in type A acute aortic dissection. J Thorac Cardiovasc Surg. 2011 141:354-60.

18. Wada S, Yamamoto S, Honda J, Hiramoto A, Wada H, Hosoda Y. Transapical aortic cannulation for cardiopulmonary bypass in type A aortic dissection operations. J Thorac Cardiovasc Surg. 2006;132:369-72.

19. Orihashi K, Ozawa M, Takahashi S, Takasaki T, Sato K, Kurosaki t, et al. Treat ment strategy for acute type A aortic dissection complicated with organ ischemia. Ann Vasc Dis. 2011;4:293-8.

Key Words: acute aortic dissection, malperfusion, early reperfusion

Readers who found these articles interesting may also like to read the following papers found in recent and future issues of our sister publications, Seminars in Thoracic and Cardiovascular Surgery and Operative Techniques in Thoracic and Cardiovascular Surgery!

\section{Adult: Aorta}

ORIGINAL SUBMISSION: Best Medical Treatment and Selective Stent-Graft Repair for Acute Type B Aortic Intramural Hematoma. Gabriele Piffaretti. Semin Thoracic Surg 2018: In press.

ORIGINAL SUBMISSION: Fluctuations in Spinal Cord Perfusion Pressure: A Harbinger of Delayed Paraplegia after Thoracoabdominal Aortic Repair. Harleen K. Sandhu. Semin Thoracic Surg 2017:451-459.

Editorial Commentary: Keep Alert Eyes on Delayed Paraplegia. Kenji Minatoya. Semin Thoracic Surg 2017:460-461.

Editorial Commentary: Problem Delayed Does Not Mean Solution Denied. Joseph S. Coselli. Semin Thoracic Surg 2017:462-463.

ORIGINAL SUBMISSION: Awake Thoracic Endovascular Aneurysm Repair for Aortic Rupture: A Case Series. Jessica Forcillo. Semin Thoracic Surg 2018:36-39.

Editorial Commentary: Awake TEVAR for Ruptured Thoracic Aneurysms: Less is More? Akiko Tanaka. Semin Thoracic Surg 2018:40-41. 